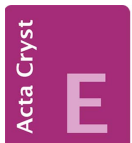

CRYSTALLOGRAPHIC COMMUNICATIONS

ISSN 2056-9890

\title{
Crystal structure of flumioxazin
}

\author{
Hyunjin Park, Jineun Kim, ${ }^{*}$ Eunjin Kwon and Tae Ho Kim*
}

Department of Chemistry and Research Institute of Natural Sciences, Gyeongsang National University, Jinju 52828, Republic of Korea. *Correspondence e-mail:

thkim@gnu.ac.kr, jekim@gnu.ac.kr

Received 8 September 2015; accepted 15 September 2015

Edited by W. T. A. Harrison, University of Aberdeen, Scotland

The title compound \{systematic name: 2-[7-fluoro-3,4-dihydro3-oxo-4-(prop-2-yn-1-yl)-2H-1,4-benzoxazin-6-yl]-4,5,6,7tetrahydro- $1 \mathrm{H}$-isoindole- $1,3(2 \mathrm{H})$-dione $\}, \mathrm{C}_{19} \mathrm{H}_{15} \mathrm{FN}_{2} \mathrm{O}_{4}$, is a dicarboximide herbicide. The dihedral angle between the maleimide and benzene ring planes is $66.13(5)^{\circ}$. In the crystal, $\mathrm{C}-\mathrm{H} \cdots \mathrm{O}$ and $\mathrm{C}-\mathrm{H} \cdots \mathrm{F}$ hydrogen bonds and weak $\mathrm{C}-$ $\mathrm{H} \cdots \pi$ interactions [3.5601 (19) $\AA$ ] link adjacent molecules, forming two-dimensional networks extending parallel to the (110) plane.

Keywords: crystal structure; dicarboximide herbicide; flumioxazin; $1 \mathrm{H}$ isoindole; 1,4-benzoxazine; $\mathrm{C}-\mathrm{H} \ldots \mathrm{F}$ hydrogen bonds.

CCDC reference: 1424397

\section{Related literature}

For information on the herbicidal properties of the title compound, see: Saladin et al. (2003); Geoffroy et al. (2004). For a related crystal structure, see: Hou et al. (2004).

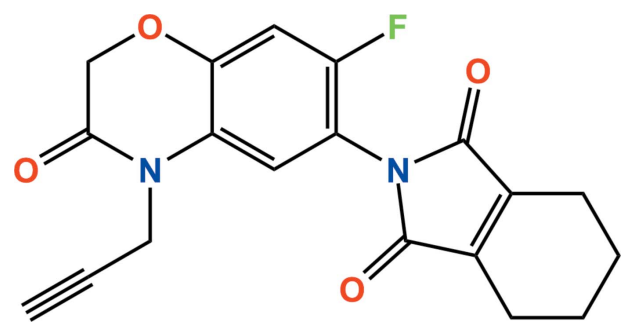

\section{Experimental}

\subsection{Crystal data}

$\mathrm{C}_{19} \mathrm{H}_{15} \mathrm{FN}_{2} \mathrm{O}_{4}$

$M_{r}=354.33$
Monoclinic, $P 2_{1} / c$ $a=8.896(1) \AA$

$$
\begin{aligned}
& b=7.1592(8) \AA \\
& c=25.708(3) \AA \\
& \beta=96.039(6)^{\circ} \\
& V=1628.2(3) \AA^{3} \\
& Z=4
\end{aligned}
$$

Mo $K \alpha$ radiation $\mu=0.11 \mathrm{~mm}^{-1}$ $T=173 \mathrm{~K}$ $0.50 \times 0.26 \times 0.05 \mathrm{~mm}$

\subsection{Data collection \\ Bruker APEXII CCD diffractometer \\ Absorption correction: multi-scan (SADABS; Bruker, 2013) \\ $T_{\min }=0.575, T_{\max }=0.746$}

\subsection{Refinement}

$R\left[F^{2}>2 \sigma\left(F^{2}\right)\right]=0.052$

$w R\left(F^{2}\right)=0.137$

$S=1.07$

4067 reflections $R_{\text {int }}=0.067$
27372 measured reflections 4067 independent reflections 3277 reflections with $I>2 \sigma(I)$

Table 1

Hydrogen-bond geometry $\left(\AA{ }^{\circ}\right)$.

$\mathrm{Cg} 1$ is the centroid of the $\mathrm{C} 3 / \mathrm{C} 4 / \mathrm{C} 8-\mathrm{C} 11$ ring.

\begin{tabular}{lllll}
\hline$D-\mathrm{H} \cdots A$ & $D-\mathrm{H}$ & $\mathrm{H} \cdots A$ & $D \cdots A$ & $D-\mathrm{H} \cdots A$ \\
\hline $\mathrm{C} 7-\mathrm{H} 7 \cdots \mathrm{O}^{\mathrm{i}}$ & 0.95 & 2.39 & $3.178(2)$ & 140 \\
$\mathrm{C} 19-\mathrm{H} 19 B \cdots \mathrm{F} 1^{\mathrm{ii}}$ & 0.99 & 2.36 & $3.289(2)$ & 155 \\
$\mathrm{C} 16-\mathrm{H} 16 A \cdots C{ }^{\mathrm{iii}}$ & 0.99 & 2.63 & $3.5601(19)$ & 157 \\
\hline
\end{tabular}

Symmetry codes: (i) $x+1, y, z$; (ii) $x, y-1, z$; (iii) $-x+2, y-\frac{1}{2},-z+\frac{1}{2}$.

Data collection: APEX2 (Bruker, 2013); cell refinement: SAINT (Bruker, 2013); data reduction: $S A I N T$; program(s) used to solve structure: SHELXS97 (Sheldrick, 2008); program(s) used to refine structure: SHELXL2013 (Sheldrick, 2015); molecular graphics: DIAMOND (Brandenburg, 2010); software used to prepare material for publication: SHELXTL (Sheldrick, 2008).

\section{Acknowledgements}

This research was supported by the Basic Science Research Program through the National Research Foundation of Korea (NRF) funded by the Ministry of Education, Science and Technology (No. 2015R1D1A4A01020317).

Supporting information for this paper is available from the IUCr electronic archives (Reference: HB7502).

\section{References}

Brandenburg, K. (2010). DIAMOND. Crystal Impact GbR, Bonn, Germany. Bruker (2013). APEX2, SAINT and SADABS. Bruker AXS Inc., Madison, Wisconsin, USA.

Geoffroy, L., Frankart, C. \& Eullaffroy, P. (2004). Environ. Pollut. 131, $233-$ 241.

Hou, K. Z., Ren, Y. G., Huang, M. Z., Song, J. \& Chen, L. G. (2004). Acta Cryst. E60, o1336-o1337.

Saladin, G., Magné, C. \& Clément, C. (2003). Chemosphere, 53, 199-206.

Sheldrick, G. M. (2008). Acta Cryst. A64, 112-122.

Sheldrick, G. M. (2015). Acta Cryst. C71, 3-8. 


\section{supporting information}

Acta Cryst. (2015). E71, o768 [doi:10.1107/S2056989015017223]

\section{Crystal structure of flumioxazin}

\section{Hyunjin Park, Jineun Kim, Eunjin Kwon and Tae Ho Kim}

\section{S1. Comment}

Flumioxazin [systematic name: 2-[7-fluoro-3,4-dihydro-3-oxo-4-(2-propyn-1-yl)-2H-1,4-benzoxazin-6-yl]-4,5,6,7-tetrahydro-1 $H$-isoindole-1,3(2H)-dione] is a soil applied pre-emergent herbicide used to inhibit development of redroot pigweed. (Geoffroy et al., 2004; Saladin et al., 2003). However, its crystal structure has not been reported until now. In the title compound (Fig. 1), the dihedral angle between pyrrole and benzne rings is $66.13(5)^{\circ}$. All bond lengths and bond angles are normal and comparable to those observed in a similar crystal structure (Hou et al., 2004).

In the crystal structure (Fig. 2), $\mathrm{C}-\mathrm{H} \cdots \mathrm{O}$ and $\mathrm{C}-\mathrm{H} \cdots \mathrm{F}$ hydrogen bonds and weak $\mathrm{C}-\mathrm{H} \cdots \pi$ interactions are observed (Table 1). Two-dimensional networks are formed by the hydrogen bonds and weak $\mathrm{C}-\mathrm{H} \cdots \pi$ interactions.

\section{S2. Experimental}

The title compound was purchased from the Dr Ehrenstorfer GmbH Company. Slow evaporation of a solution in $\mathrm{CH}_{3} \mathrm{CN}$ gave single crystals suitable for X-ray analysis.

\section{S3. Refinement}

All H-atoms were positioned geometrically and refined using a riding model with $\mathrm{d}(\mathrm{C}-\mathrm{H})=0.99 \AA, U_{\text {iso }}=1.2 U_{\text {eq }}(\mathrm{C})$ for $\mathrm{CH}_{2}$ group, $\mathrm{d}(\mathrm{C}-\mathrm{H})=0.95 \AA, U_{\text {iso }}=1.2 U_{\text {eq }}(\mathrm{C})$ for aromatic $\mathrm{C}-\mathrm{H}$ aromatic $\mathrm{C}-\mathrm{H}$ and $\mathrm{C} s p-\mathrm{H}$.

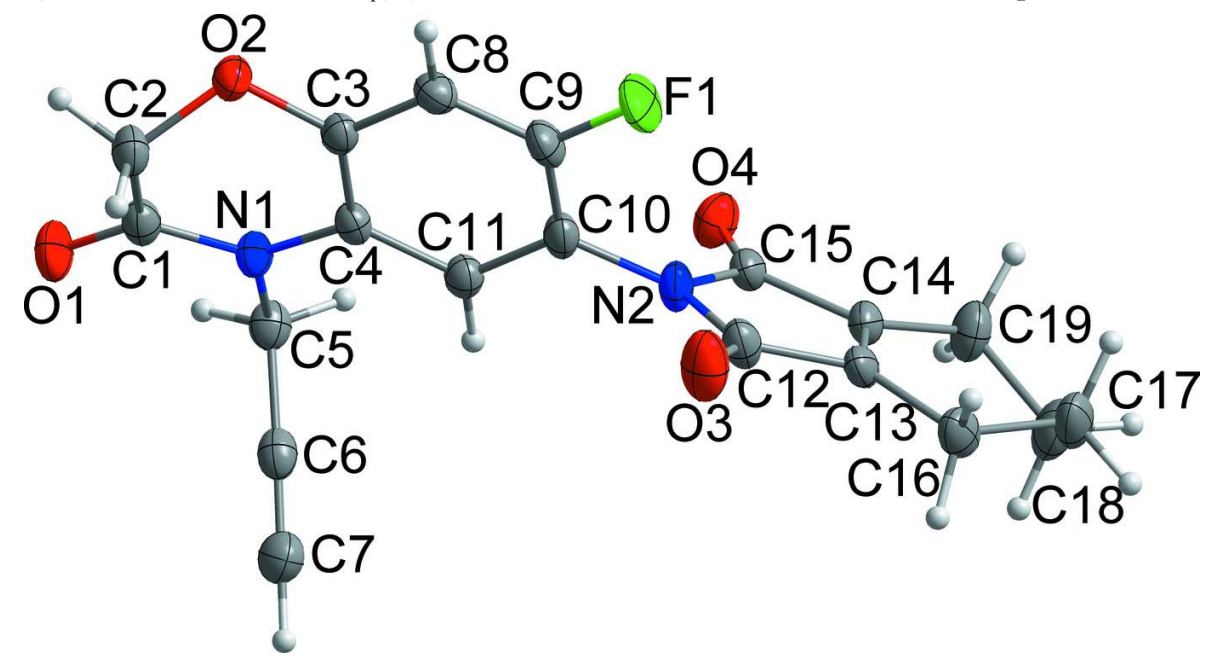

\section{Figure 1}

The asymmetric unit of the title compound with the atom-numbering scheme. Displacement ellipsoids are drawn at the $50 \%$ probability level. $\mathrm{H}$ atoms are shown as small spheres of arbitrary radius. 


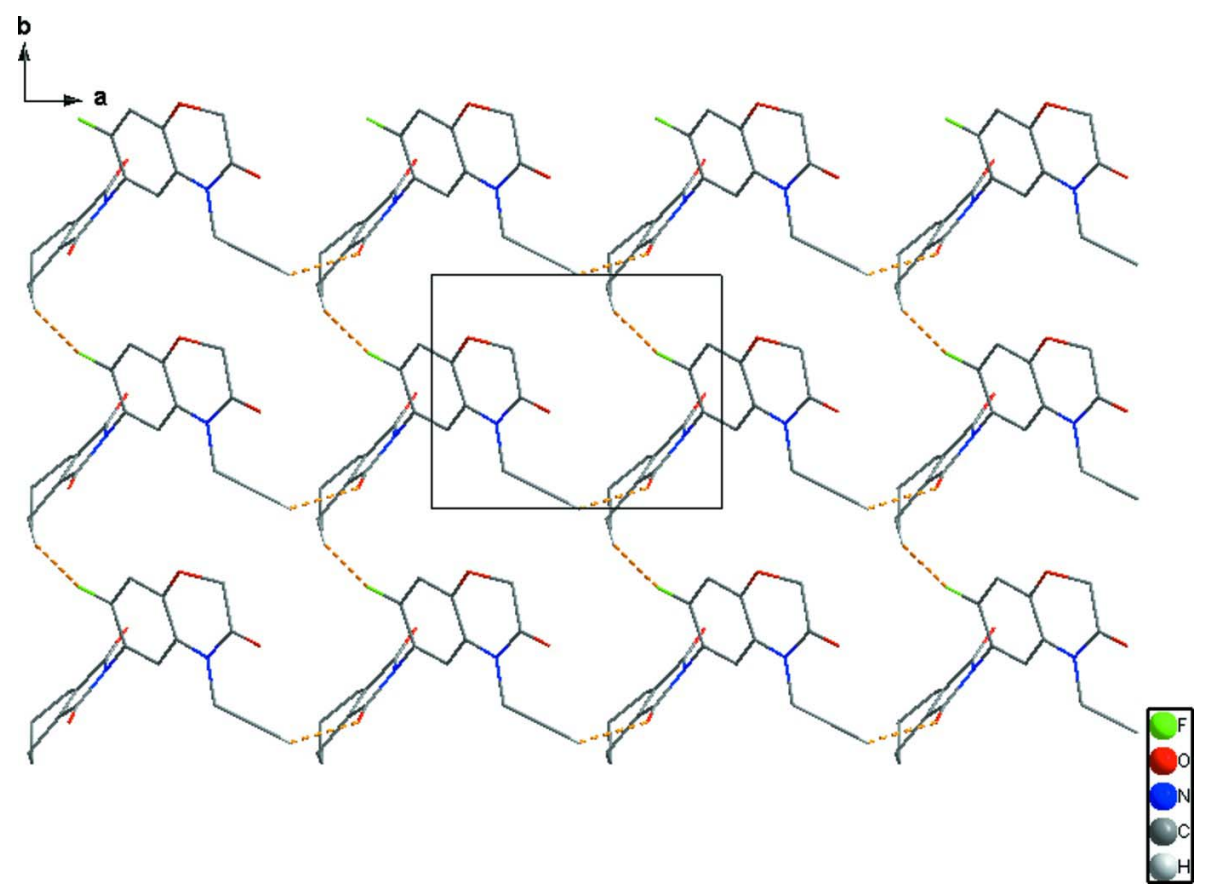

Figure 2

Crystal packing viewed along the $c$ axis. The intermolecular interactions are shown as dashed lines.

2-[7-Fluoro-3,4-dihydro-3-oxo-4-(prop-2-yn-1-yl)-2H-1,4-benzoxazin-6-yl]-4,5,6,7-tetrahydro- $1 H$ isoindole-1,3(2H)-dione

Crystal data

$\mathrm{C}_{19} \mathrm{H}_{15} \mathrm{FN}_{2} \mathrm{O}_{4}$

$M_{r}=354.33$

Monoclinic, $P 2_{1} / c$

$a=8.896(1) \AA$

$b=7.1592(8) \AA$

$c=25.708(3) \AA$

$\beta=96.039(6)^{\circ}$

$V=1628.2(3) \AA^{3}$

$Z=4$

Data collection

Bruker APEXII CCD

diffractometer

$\varphi$ and $\omega$ scans

Absorption correction: multi-scan

(SADABS; Bruker, 2013)

$T_{\text {min }}=0.575, T_{\max }=0.746$

27372 measured reflections

Refinement

Refinement on $F^{2}$

Least-squares matrix: full

$R\left[F^{2}>2 \sigma\left(F^{2}\right)\right]=0.052$

$w R\left(F^{2}\right)=0.137$
$F(000)=736$

$D_{\mathrm{x}}=1.445 \mathrm{Mg} \mathrm{m}^{-3}$

Mo $K \alpha$ radiation, $\lambda=0.71073 \AA$

Cell parameters from 8497 reflections

$\theta=2.7-28.2^{\circ}$

$\mu=0.11 \mathrm{~mm}^{-1}$

$T=173 \mathrm{~K}$

Plate, colourless

$0.50 \times 0.26 \times 0.05 \mathrm{~mm}$

4067 independent reflections

3277 reflections with $I>2 \sigma(I)$

$R_{\text {int }}=0.067$

$\theta_{\max }=28.4^{\circ}, \theta_{\min }=1.6^{\circ}$

$h=-11 \rightarrow 11$

$k=-9 \rightarrow 9$

$l=-34 \rightarrow 34$

$S=1.07$

4067 reflections

235 parameters

0 restraints 
Hydrogen site location: inferred from neighbouring sites

$\mathrm{H}$-atom parameters constrained

$$
\begin{aligned}
& w=1 /\left[\sigma^{2}\left(F_{\mathrm{o}}^{2}\right)+(0.0535 P)^{2}+0.9028 P\right] \\
& \text { where } P=\left(F_{\mathrm{o}}^{2}+2 F_{\mathrm{c}}^{2}\right) / 3 \\
& (\Delta / \sigma)_{\max }<0.001 \\
& \Delta \rho_{\max }=0.32 \mathrm{e} \AA^{-3} \\
& \Delta \rho_{\min }=-0.27 \mathrm{e} \AA^{-3}
\end{aligned}
$$

Special details

Geometry. All e.s.d.'s (except the e.s.d. in the dihedral angle between two 1.s. planes) are estimated using the full

\begin{tabular}{|c|c|c|c|c|}
\hline & $x$ & $y$ & $z$ & $U_{\text {iso }} * / U_{\text {eq }}$ \\
\hline $\mathrm{F} 1$ & $0.77768(12)$ & $0.66568(18)$ & $0.33755(5)$ & $0.0431(3)$ \\
\hline O1 & $1.40190(15)$ & 0.41585 (19) & $0.54636(5)$ & $0.0359(3)$ \\
\hline $\mathrm{O} 2$ & $1.12467(13)$ & $0.73087(17)$ & $0.48786(4)$ & $0.0273(3)$ \\
\hline $\mathrm{O} 3$ & $0.94166(16)$ & $0.4892(2)$ & $0.25135(5)$ & $0.0459(4)$ \\
\hline $\mathrm{O} 4$ & $0.74322(15)$ & $0.08897(18)$ & $0.36415(5)$ & $0.0331(3)$ \\
\hline N1 & $1.22083(15)$ & $0.3652(2)$ & $0.47885(5)$ & $0.0235(3)$ \\
\hline N2 & $0.86942(16)$ & $0.3094(2)$ & $0.31951(5)$ & $0.0276(3)$ \\
\hline $\mathrm{C} 1$ & $1.30559(18)$ & $0.4767(3)$ & $0.51371(6)$ & $0.0265(4)$ \\
\hline $\mathrm{C} 2$ & $1.27631(19)$ & 0.6824 & $0.50845(7)$ & $0.0291(4)$ \\
\hline $\mathrm{H} 2 \mathrm{~A}$ & 1.2983 & 0.7410 & 0.5433 & $0.035^{*}$ \\
\hline $\mathrm{H} 2 \mathrm{~B}$ & 1.3474 & 0.7360 & 0.4853 & $0.035^{*}$ \\
\hline $\mathrm{C} 3$ & $1.06753(17)$ & $0.6259(2)$ & $0.44577(6)$ & $0.0226(3)$ \\
\hline $\mathrm{C} 4$ & $1.11458(17)$ & $0.4423(2)$ & $0.43988(6)$ & $0.0217(3)$ \\
\hline $\mathrm{C} 5$ & $1.2461(2)$ & $0.1636(2)$ & $0.48166(6)$ & $0.0272(4)$ \\
\hline $\mathrm{H} 5 \mathrm{~A}$ & 1.1481 & 0.0987 & 0.4739 & $0.033 *$ \\
\hline H5B & 1.2872 & 0.1300 & 0.5177 & $0.033^{*}$ \\
\hline C6 & $1.3508(2)$ & $0.0983(2)$ & $0.44508(7)$ & $0.0284(4)$ \\
\hline $\mathrm{C} 7$ & $1.4371(2)$ & $0.0447(3)$ & $0.41648(7)$ & $0.0357(4)$ \\
\hline $\mathrm{H7}$ & 1.5064 & 0.0015 & 0.3935 & $0.043 *$ \\
\hline $\mathrm{C} 8$ & $0.95463(18)$ & $0.7036(3)$ & $0.41121(6)$ & $0.0266(4)$ \\
\hline H8 & 0.9225 & 0.8288 & 0.4154 & $0.032 *$ \\
\hline $\mathrm{C} 9$ & $0.89012(18)$ & $0.5945(3)$ & $0.37066(7)$ & $0.0284(4)$ \\
\hline $\mathrm{C} 10$ & $0.93760(18)$ & $0.4142(3)$ & $0.36273(6)$ & $0.0261(4)$ \\
\hline C11 & $1.05195(18)$ & $0.3384(2)$ & $0.39736(6)$ & $0.0244(3)$ \\
\hline H11 & 1.0873 & 0.2154 & 0.3920 & $0.029 *$ \\
\hline $\mathrm{C} 12$ & 0.87139 (19) & $0.3604(3)$ & $0.26659(7)$ & $0.0298(4)$ \\
\hline $\mathrm{C} 13$ & $0.76914(17)$ & $0.2264(3)$ & $0.23609(6)$ & $0.0252(4)$ \\
\hline $\mathrm{C} 14$ & $0.70932(18)$ & $0.1121(2)$ & $0.26880(6)$ & $0.0246(4)$ \\
\hline $\mathrm{C} 15$ & $0.77039(17)$ & $0.1593(2)$ & $0.32346(6)$ & $0.0235(3)$ \\
\hline $\mathrm{C} 16$ & $0.7429(2)$ & 0.2189 & $0.17802(6)$ & $0.0338(4)$ \\
\hline H16A & 0.8355 & 0.1746 & 0.1637 & $0.041 *$ \\
\hline H16B & 0.7193 & 0.3457 & 0.1640 & $0.041^{*}$ \\
\hline C17 & $0.6125(2)$ & 0.0878 & $0.16111(7)$ & $0.0430(5)$ \\
\hline H17A & 0.5158 & 0.1542 & 0.1634 & $0.052 *$ \\
\hline
\end{tabular}
covariance matrix. The cell e.s.d.'s are taken into account individually in the estimation of e.s.d.'s in distances, angles and torsion angles; correlations between e.s.d.'s in cell parameters are only used when they are defined by crystal symmetry. An approximate (isotropic) treatment of cell e.s.d.'s is used for estimating e.s.d.'s involving l.s. planes.

Fractional atomic coordinates and isotropic or equivalent isotropic displacement parameters $\left(\AA^{2}\right)$ 
supporting information

\begin{tabular}{lllll} 
H17B & 0.6171 & 0.0519 & 0.1241 & $0.052^{*}$ \\
C18 & $0.6158(3)$ & $-0.0865(3)$ & $0.19432(8)$ & $0.0468(5)$ \\
H18A & 0.7113 & -0.1546 & 0.1912 & $0.056^{*}$ \\
H18B & 0.5311 & -0.1690 & 0.1809 & $0.056^{*}$ \\
C19 & $0.6033(2)$ & $-0.0436(3)$ & $0.25236(7)$ & $0.0361(4)$ \\
H19A & 0.4984 & -0.0070 & 0.2573 & $0.043^{*}$ \\
H19B & 0.6297 & -0.1559 & 0.2739 & $0.043^{*}$ \\
\hline
\end{tabular}

Atomic displacement parameters $\left(\AA^{2}\right)$

\begin{tabular}{lllllll}
\hline & $U^{11}$ & $U^{22}$ & $U^{33}$ & $U^{12}$ & $U^{13}$ & $U^{23}$ \\
\hline $\mathrm{F} 1$ & $0.0319(6)$ & $0.0490(7)$ & $0.0443(6)$ & $0.0126(5)$ & $-0.0160(5)$ & $-0.0011(5)$ \\
$\mathrm{O} 1$ & $0.0353(7)$ & $0.0380(8)$ & $0.0310(6)$ & $-0.0010(6)$ & $-0.0128(5)$ & $0.0025(6)$ \\
$\mathrm{O} 2$ & $0.0270(6)$ & $0.0264(6)$ & $0.0273(6)$ & $-0.0010(5)$ & $-0.0021(5)$ & $-0.0052(5)$ \\
O3 & $0.0433(8)$ & $0.0572(10)$ & $0.0362(7)$ & $-0.0243(7)$ & $-0.0003(6)$ & $0.0082(7)$ \\
O4 & $0.0408(7)$ & $0.0352(7)$ & $0.0228(6)$ & $-0.0063(6)$ & $0.0011(5)$ & $0.0015(5)$ \\
N1 & $0.0241(7)$ & $0.0220(7)$ & $0.0230(6)$ & $0.0000(6)$ & $-0.0034(5)$ & $0.0015(5)$ \\
N2 & $0.0235(7)$ & $0.0368(8)$ & $0.0213(6)$ & $-0.0068(6)$ & $-0.0036(5)$ & $-0.0004(6)$ \\
C1 & $0.0234(8)$ & $0.0334(9)$ & $0.0222(7)$ & $-0.0031(7)$ & $-0.0004(6)$ & $0.0009(7)$ \\
C2 & $0.0264(8)$ & $0.0290(9)$ & $0.0301(8)$ & $-0.0042(7)$ & $-0.0055(7)$ & $-0.0026(7)$ \\
C3 & $0.0197(7)$ & $0.0258(8)$ & $0.0222(7)$ & $-0.0031(6)$ & $0.0012(6)$ & $-0.0014(6)$ \\
C4 & $0.0174(7)$ & $0.0247(8)$ & $0.0224(7)$ & $-0.0019(6)$ & $0.0001(6)$ & $0.0022(6)$ \\
C5 & $0.0296(8)$ & $0.0247(9)$ & $0.0263(8)$ & $-0.0005(7)$ & $-0.0016(6)$ & $0.0027(7)$ \\
C6 & $0.0288(8)$ & $0.0234(9)$ & $0.0309(8)$ & $-0.0005(7)$ & $-0.0065(7)$ & $0.0005(7)$ \\
C7 & $0.0341(10)$ & $0.0369(11)$ & $0.0354(9)$ & $-0.0024(8)$ & $0.0004(8)$ & $-0.0058(8)$ \\
C8 & $0.0221(8)$ & $0.0269(9)$ & $0.0306(8)$ & $0.0032(7)$ & $0.0020(6)$ & $0.0001(7)$ \\
C9 & $0.0196(8)$ & $0.0354(10)$ & $0.0289(8)$ & $0.0028(7)$ & $-0.0041(6)$ & $0.0036(7)$ \\
C10 & $0.0208(8)$ & $0.0332(9)$ & $0.0235(7)$ & $-0.0050(7)$ & $-0.0018(6)$ & $-0.0023(7)$ \\
C11 & $0.0220(8)$ & $0.0263(8)$ & $0.0245(7)$ & $-0.0026(7)$ & $0.0007(6)$ & $-0.0008(7)$ \\
C12 & $0.0221(8)$ & $0.0410(10)$ & $0.0255(8)$ & $-0.0046(8)$ & $-0.0010(6)$ & $0.0024(7)$ \\
C13 & $0.0176(7)$ & $0.0355(9)$ & $0.0217(7)$ & $0.0024(7)$ & $-0.0022(6)$ & $-0.0006(7)$ \\
C14 & $0.0204(7)$ & $0.0300(9)$ & $0.0221(7)$ & $0.0014(7)$ & $-0.0031(6)$ & $-0.0020(6)$ \\
C15 & $0.0192(7)$ & $0.0280(8)$ & $0.0226(7)$ & $0.0017(6)$ & $-0.0013(6)$ & $-0.0010(6)$ \\
C16 & $0.0285(9)$ & $0.0515(12)$ & $0.0207(8)$ & $0.0011(8)$ & $-0.0011(6)$ & $0.0025(8)$ \\
C17 & $0.0322(10)$ & $0.0718(15)$ & $0.0234(8)$ & $-0.0059(10)$ & $-0.0039(7)$ & $-0.0085(9)$ \\
C18 & $0.0483(12)$ & $0.0536(14)$ & $0.0368(10)$ & $-0.0122(10)$ & $-0.0032(9)$ & $-0.0155(10)$ \\
C19 & $0.0397(10)$ & $0.0367(11)$ & $0.0301(9)$ & $-0.0123(9)$ & $-0.0047(7)$ & $-0.0025(8)$ \\
& & & & & & \\
\hline & & & & & & \\
\end{tabular}

Geometric parameters $\left(\hat{A},{ }^{o}\right)$

\begin{tabular}{llll}
\hline $\mathrm{F} 1-\mathrm{C} 9$ & $1.3436(19)$ & $\mathrm{C} 7-\mathrm{H} 7$ & 0.9500 \\
$\mathrm{O} 1-\mathrm{C} 1$ & $1.215(2)$ & $\mathrm{C} 8-\mathrm{C} 9$ & $1.378(2)$ \\
$\mathrm{O} 2-\mathrm{C} 3$ & $1.3707(19)$ & $\mathrm{C} 8-\mathrm{H} 8$ & 0.9500 \\
$\mathrm{O} 2-\mathrm{C} 2$ & $1.439(2)$ & $\mathrm{C} 9-\mathrm{C} 10$ & $1.380(3)$ \\
$\mathrm{O} 3-\mathrm{C} 12$ & $1.203(2)$ & $\mathrm{C} 10-\mathrm{C} 11$ & $1.390(2)$ \\
$\mathrm{O} 4-\mathrm{C} 15$ & $1.208(2)$ & $\mathrm{C} 11-\mathrm{H} 11$ & 0.9500 \\
$\mathrm{~N} 1-\mathrm{C} 1$ & $1.366(2)$ & $\mathrm{C} 12-\mathrm{C} 13$ & $1.487(2)$ \\
$\mathrm{N} 1-\mathrm{C} 4$ & $1.4153(19)$ & $\mathrm{C} 13-\mathrm{C} 14$ & $1.325(2)$
\end{tabular}




\begin{tabular}{|c|c|c|c|}
\hline $\mathrm{N} 1-\mathrm{C} 5$ & $1.461(2)$ & $\mathrm{C} 13-\mathrm{C} 16$ & $1.488(2)$ \\
\hline $\mathrm{N} 2-\mathrm{C} 15$ & $1.400(2)$ & $\mathrm{C} 14-\mathrm{C} 15$ & $1.491(2)$ \\
\hline $\mathrm{N} 2-\mathrm{C} 12$ & $1.410(2)$ & $\mathrm{C} 14-\mathrm{C} 19$ & $1.493(2)$ \\
\hline $\mathrm{N} 2-\mathrm{C} 10$ & $1.422(2)$ & $\mathrm{C} 16-\mathrm{C} 17$ & $1.520(3)$ \\
\hline $\mathrm{C} 1-\mathrm{C} 2$ & $1.499(3)$ & C16-H16A & 0.9900 \\
\hline $\mathrm{C} 2-\mathrm{H} 2 \mathrm{~A}$ & 0.9900 & C16-H16B & 0.9900 \\
\hline $\mathrm{C} 2-\mathrm{H} 2 \mathrm{~B}$ & 0.9900 & $\mathrm{C} 17-\mathrm{C} 18$ & $1.511(3)$ \\
\hline $\mathrm{C} 3-\mathrm{C} 8$ & $1.386(2)$ & C17-H17A & 0.9900 \\
\hline $\mathrm{C} 3-\mathrm{C} 4$ & $1.392(2)$ & C17-H17B & 0.9900 \\
\hline $\mathrm{C} 4-\mathrm{C} 11$ & $1.390(2)$ & $\mathrm{C} 18-\mathrm{C} 19$ & $1.539(3)$ \\
\hline $\mathrm{C} 5-\mathrm{C} 6$ & $1.468(2)$ & $\mathrm{C} 18-\mathrm{H} 18 \mathrm{~A}$ & 0.9900 \\
\hline $\mathrm{C} 5-\mathrm{H} 5 \mathrm{~A}$ & 0.9900 & $\mathrm{C} 18-\mathrm{H} 18 \mathrm{~B}$ & 0.9900 \\
\hline C5-H5B & 0.9900 & C19-H19A & 0.9900 \\
\hline $\mathrm{C} 6-\mathrm{C} 7$ & $1.182(3)$ & C19-H19B & 0.9900 \\
\hline $\mathrm{C} 3-\mathrm{O} 2-\mathrm{C} 2$ & $114.43(13)$ & $\mathrm{C} 4-\mathrm{C} 11-\mathrm{C} 10$ & $120.04(16)$ \\
\hline $\mathrm{C} 1-\mathrm{N} 1-\mathrm{C} 4$ & $121.20(14)$ & $\mathrm{C} 4-\mathrm{C} 11-\mathrm{H} 11$ & 120.0 \\
\hline $\mathrm{C} 1-\mathrm{N} 1-\mathrm{C} 5$ & $118.24(14)$ & $\mathrm{C} 10-\mathrm{C} 11-\mathrm{H} 11$ & 120.0 \\
\hline $\mathrm{C} 4-\mathrm{N} 1-\mathrm{C} 5$ & $120.54(13)$ & $\mathrm{O} 3-\mathrm{C} 12-\mathrm{N} 2$ & $124.88(16)$ \\
\hline $\mathrm{C} 15-\mathrm{N} 2-\mathrm{C} 12$ & $109.88(13)$ & $\mathrm{O} 3-\mathrm{C} 12-\mathrm{C} 13$ & $129.26(16)$ \\
\hline $\mathrm{C} 15-\mathrm{N} 2-\mathrm{C} 10$ & $124.61(14)$ & $\mathrm{N} 2-\mathrm{C} 12-\mathrm{C} 13$ & $105.84(14)$ \\
\hline $\mathrm{C} 12-\mathrm{N} 2-\mathrm{C} 10$ & $124.73(15)$ & $\mathrm{C} 14-\mathrm{C} 13-\mathrm{C} 12$ & 109.15 (14) \\
\hline $\mathrm{O} 1-\mathrm{C} 1-\mathrm{N} 1$ & $122.97(17)$ & $\mathrm{C} 14-\mathrm{C} 13-\mathrm{C} 16$ & $125.71(16)$ \\
\hline $\mathrm{O} 1-\mathrm{C} 1-\mathrm{C} 2$ & $121.16(16)$ & $\mathrm{C} 12-\mathrm{C} 13-\mathrm{C} 16$ & $125.12(16)$ \\
\hline $\mathrm{N} 1-\mathrm{C} 1-\mathrm{C} 2$ & $115.85(14)$ & $\mathrm{C} 13-\mathrm{C} 14-\mathrm{C} 15$ & $109.11(15)$ \\
\hline $\mathrm{O} 2-\mathrm{C} 2-\mathrm{C} 1$ & $114.67(14)$ & $\mathrm{C} 13-\mathrm{C} 14-\mathrm{C} 19$ & $124.47(15)$ \\
\hline $\mathrm{O} 2-\mathrm{C} 2-\mathrm{H} 2 \mathrm{~A}$ & 108.6 & $\mathrm{C} 15-\mathrm{C} 14-\mathrm{C} 19$ & $126.37(15)$ \\
\hline $\mathrm{C} 1-\mathrm{C} 2-\mathrm{H} 2 \mathrm{~A}$ & 108.6 & $\mathrm{O} 4-\mathrm{C} 15-\mathrm{N} 2$ & $124.57(15)$ \\
\hline $\mathrm{O} 2-\mathrm{C} 2-\mathrm{H} 2 \mathrm{~B}$ & 108.6 & $\mathrm{O} 4-\mathrm{C} 15-\mathrm{C} 14$ & $129.44(16)$ \\
\hline $\mathrm{C} 1-\mathrm{C} 2-\mathrm{H} 2 \mathrm{~B}$ & 108.6 & $\mathrm{~N} 2-\mathrm{C} 15-\mathrm{C} 14$ & $105.98(13)$ \\
\hline $\mathrm{H} 2 \mathrm{~A}-\mathrm{C} 2-\mathrm{H} 2 \mathrm{~B}$ & 107.6 & $\mathrm{C} 13-\mathrm{C} 16-\mathrm{C} 17$ & $110.12(15)$ \\
\hline $\mathrm{O} 2-\mathrm{C} 3-\mathrm{C} 8$ & $117.99(15)$ & $\mathrm{C} 13-\mathrm{C} 16-\mathrm{H} 16 \mathrm{~A}$ & 109.6 \\
\hline $\mathrm{O} 2-\mathrm{C} 3-\mathrm{C} 4$ & $120.85(14)$ & $\mathrm{C} 17-\mathrm{C} 16-\mathrm{H} 16 \mathrm{~A}$ & 109.6 \\
\hline $\mathrm{C} 8-\mathrm{C} 3-\mathrm{C} 4$ & $121.02(15)$ & $\mathrm{C} 13-\mathrm{C} 16-\mathrm{H} 16 \mathrm{~B}$ & 109.6 \\
\hline $\mathrm{C} 11-\mathrm{C} 4-\mathrm{C} 3$ & $119.39(14)$ & $\mathrm{C} 17-\mathrm{C} 16-\mathrm{H} 16 \mathrm{~B}$ & 109.6 \\
\hline $\mathrm{C} 11-\mathrm{C} 4-\mathrm{N} 1$ & $122.06(15)$ & $\mathrm{H} 16 \mathrm{~A}-\mathrm{C} 16-\mathrm{H} 16 \mathrm{~B}$ & 108.2 \\
\hline $\mathrm{C} 3-\mathrm{C} 4-\mathrm{N} 1$ & $118.50(14)$ & $\mathrm{C} 18-\mathrm{C} 17-\mathrm{C} 16$ & $112.33(16)$ \\
\hline $\mathrm{N} 1-\mathrm{C} 5-\mathrm{C} 6$ & $112.80(14)$ & $\mathrm{C} 18-\mathrm{C} 17-\mathrm{H} 17 \mathrm{~A}$ & 109.1 \\
\hline $\mathrm{N} 1-\mathrm{C} 5-\mathrm{H} 5 \mathrm{~A}$ & 109.0 & $\mathrm{C} 16-\mathrm{C} 17-\mathrm{H} 17 \mathrm{~A}$ & 109.1 \\
\hline $\mathrm{C} 6-\mathrm{C} 5-\mathrm{H} 5 \mathrm{~A}$ & 109.0 & $\mathrm{C} 18-\mathrm{C} 17-\mathrm{H} 17 \mathrm{~B}$ & 109.1 \\
\hline $\mathrm{N} 1-\mathrm{C} 5-\mathrm{H} 5 \mathrm{~B}$ & 109.0 & $\mathrm{C} 16-\mathrm{C} 17-\mathrm{H} 17 \mathrm{~B}$ & 109.1 \\
\hline $\mathrm{C} 6-\mathrm{C} 5-\mathrm{H} 5 \mathrm{~B}$ & 109.0 & $\mathrm{H} 17 \mathrm{~A}-\mathrm{C} 17-\mathrm{H} 17 \mathrm{~B}$ & 107.9 \\
\hline $\mathrm{H} 5 \mathrm{~A}-\mathrm{C} 5-\mathrm{H} 5 \mathrm{~B}$ & 107.8 & $\mathrm{C} 17-\mathrm{C} 18-\mathrm{C} 19$ & $112.57(18)$ \\
\hline $\mathrm{C} 7-\mathrm{C} 6-\mathrm{C} 5$ & $178.63(19)$ & $\mathrm{C} 17-\mathrm{C} 18-\mathrm{H} 18 \mathrm{~A}$ & 109.1 \\
\hline $\mathrm{C} 6-\mathrm{C} 7-\mathrm{H} 7$ & 180.0 & $\mathrm{C} 19-\mathrm{C} 18-\mathrm{H} 18 \mathrm{~A}$ & 109.1 \\
\hline $\mathrm{C} 9-\mathrm{C} 8-\mathrm{C} 3$ & $118.25(16)$ & $\mathrm{C} 17-\mathrm{C} 18-\mathrm{H} 18 \mathrm{~B}$ & 109.1 \\
\hline $\mathrm{C} 9-\mathrm{C} 8-\mathrm{H} 8$ & 120.9 & $\mathrm{C} 19-\mathrm{C} 18-\mathrm{H} 18 \mathrm{~B}$ & 109.1 \\
\hline $\mathrm{C} 3-\mathrm{C} 8-\mathrm{H} 8$ & 120.9 & $\mathrm{H} 18 \mathrm{~A}-\mathrm{C} 18-\mathrm{H} 18 \mathrm{~B}$ & 107.8 \\
\hline
\end{tabular}




\begin{tabular}{|c|c|c|c|}
\hline $\mathrm{F} 1-\mathrm{C} 9-\mathrm{C} 8$ & $119.17(16)$ & $\mathrm{C} 14-\mathrm{C} 19-\mathrm{C} 18$ & $108.39(16)$ \\
\hline $\mathrm{F} 1-\mathrm{C} 9-\mathrm{C} 10$ & $118.68(15)$ & $\mathrm{C} 14-\mathrm{C} 19-\mathrm{H} 19 \mathrm{~A}$ & 110.0 \\
\hline $\mathrm{C} 8-\mathrm{C} 9-\mathrm{C} 10$ & $122.15(15)$ & $\mathrm{C} 18-\mathrm{C} 19-\mathrm{H} 19 \mathrm{~A}$ & 110.0 \\
\hline $\mathrm{C} 9-\mathrm{C} 10-\mathrm{C} 11$ & $119.04(15)$ & $\mathrm{C} 14-\mathrm{C} 19-\mathrm{H} 19 \mathrm{~B}$ & 110.0 \\
\hline $\mathrm{C} 9-\mathrm{C} 10-\mathrm{N} 2$ & $119.79(15)$ & $\mathrm{C} 18-\mathrm{C} 19-\mathrm{H} 19 \mathrm{~B}$ & 110.0 \\
\hline $\mathrm{C} 11-\mathrm{C} 10-\mathrm{N} 2$ & $121.17(16)$ & $\mathrm{H} 19 \mathrm{~A}-\mathrm{C} 19-\mathrm{H} 19 \mathrm{~B}$ & 108.4 \\
\hline $\mathrm{C} 4-\mathrm{N} 1-\mathrm{C} 1-\mathrm{O} 1$ & $-177.07(15)$ & $\mathrm{C} 3-\mathrm{C} 4-\mathrm{C} 11-\mathrm{C} 10$ & $-3.5(2)$ \\
\hline $\mathrm{C} 5-\mathrm{N} 1-\mathrm{C} 1-\mathrm{O} 1$ & $0.9(2)$ & $\mathrm{N} 1-\mathrm{C} 4-\mathrm{C} 11-\mathrm{C} 10$ & $173.89(14)$ \\
\hline $\mathrm{C} 4-\mathrm{N} 1-\mathrm{C} 1-\mathrm{C} 2$ & $1.1(2)$ & $\mathrm{C} 9-\mathrm{C} 10-\mathrm{C} 11-\mathrm{C} 4$ & $1.4(2)$ \\
\hline $\mathrm{C} 5-\mathrm{N} 1-\mathrm{C} 1-\mathrm{C} 2$ & $179.13(15)$ & $\mathrm{N} 2-\mathrm{C} 10-\mathrm{C} 11-\mathrm{C} 4$ & $-178.70(15)$ \\
\hline $\mathrm{C} 3-\mathrm{O} 2-\mathrm{C} 2-\mathrm{C} 1$ & $-44.1(2)$ & $\mathrm{C} 15-\mathrm{N} 2-\mathrm{C} 12-\mathrm{O} 3$ & $177.05(18)$ \\
\hline $\mathrm{O} 1-\mathrm{C} 1-\mathrm{C} 2-\mathrm{O} 2$ & $-152.56(16)$ & $\mathrm{C} 10-\mathrm{N} 2-\mathrm{C} 12-\mathrm{O} 3$ & $6.8(3)$ \\
\hline $\mathrm{N} 1-\mathrm{C} 1-\mathrm{C} 2-\mathrm{O} 2$ & $29.2(2)$ & $\mathrm{C} 15-\mathrm{N} 2-\mathrm{C} 12-\mathrm{C} 13$ & $-1.89(19)$ \\
\hline $\mathrm{C} 2-\mathrm{O} 2-\mathrm{C} 3-\mathrm{C} 8$ & $-154.71(15)$ & $\mathrm{C} 10-\mathrm{N} 2-\mathrm{C} 12-\mathrm{C} 13$ & $-172.15(15)$ \\
\hline $\mathrm{C} 2-\mathrm{O} 2-\mathrm{C} 3-\mathrm{C} 4$ & $29.4(2)$ & $\mathrm{O} 3-\mathrm{C} 12-\mathrm{C} 13-\mathrm{C} 14$ & $-176.9(2)$ \\
\hline $\mathrm{O} 2-\mathrm{C} 3-\mathrm{C} 4-\mathrm{C} 11$ & $178.59(14)$ & $\mathrm{N} 2-\mathrm{C} 12-\mathrm{C} 13-\mathrm{C} 14$ & $1.96(19)$ \\
\hline $\mathrm{C} 8-\mathrm{C} 3-\mathrm{C} 4-\mathrm{C} 11$ & $2.9(2)$ & $\mathrm{O} 3-\mathrm{C} 12-\mathrm{C} 13-\mathrm{C} 16$ & $5.0(3)$ \\
\hline $\mathrm{O} 2-\mathrm{C} 3-\mathrm{C} 4-\mathrm{N} 1$ & $1.1(2)$ & $\mathrm{N} 2-\mathrm{C} 12-\mathrm{C} 13-\mathrm{C} 16$ & $-176.16(16)$ \\
\hline $\mathrm{C} 8-\mathrm{C} 3-\mathrm{C} 4-\mathrm{N} 1$ & $-174.58(14)$ & $\mathrm{C} 12-\mathrm{C} 13-\mathrm{C} 14-\mathrm{C} 15$ & $-1.26(19)$ \\
\hline $\mathrm{C} 1-\mathrm{N} 1-\mathrm{C} 4-\mathrm{C} 11$ & $165.50(15)$ & $\mathrm{C} 16-\mathrm{C} 13-\mathrm{C} 14-\mathrm{C} 15$ & $176.84(16)$ \\
\hline $\mathrm{C} 5-\mathrm{N} 1-\mathrm{C} 4-\mathrm{C} 11$ & $-12.5(2)$ & $\mathrm{C} 12-\mathrm{C} 13-\mathrm{C} 14-\mathrm{C} 19$ & $-178.74(16)$ \\
\hline $\mathrm{C} 1-\mathrm{N} 1-\mathrm{C} 4-\mathrm{C} 3$ & $-17.1(2)$ & $\mathrm{C} 16-\mathrm{C} 13-\mathrm{C} 14-\mathrm{C} 19$ & $-0.6(3)$ \\
\hline $\mathrm{C} 5-\mathrm{N} 1-\mathrm{C} 4-\mathrm{C} 3$ & $164.92(15)$ & $\mathrm{C} 12-\mathrm{N} 2-\mathrm{C} 15-\mathrm{O} 4$ & $-178.20(17)$ \\
\hline $\mathrm{C} 1-\mathrm{N} 1-\mathrm{C} 5-\mathrm{C} 6$ & $-95.61(17)$ & $\mathrm{C} 10-\mathrm{N} 2-\mathrm{C} 15-\mathrm{O} 4$ & $-7.9(3)$ \\
\hline $\mathrm{C} 4-\mathrm{N} 1-\mathrm{C} 5-\mathrm{C} 6$ & $82.40(19)$ & $\mathrm{C} 12-\mathrm{N} 2-\mathrm{C} 15-\mathrm{C} 14$ & $1.17(18)$ \\
\hline $\mathrm{O} 2-\mathrm{C} 3-\mathrm{C} 8-\mathrm{C} 9$ & $-176.00(15)$ & $\mathrm{C} 10-\mathrm{N} 2-\mathrm{C} 15-\mathrm{C} 14$ & $171.45(15)$ \\
\hline $\mathrm{C} 4-\mathrm{C} 3-\mathrm{C} 8-\mathrm{C} 9$ & $-0.2(2)$ & $\mathrm{C} 13-\mathrm{C} 14-\mathrm{C} 15-\mathrm{O} 4$ & $179.43(18)$ \\
\hline $\mathrm{C} 3-\mathrm{C} 8-\mathrm{C} 9-\mathrm{F} 1$ & $178.24(15)$ & $\mathrm{C} 19-\mathrm{C} 14-\mathrm{C} 15-\mathrm{O} 4$ & $-3.2(3)$ \\
\hline $\mathrm{C} 3-\mathrm{C} 8-\mathrm{C} 9-\mathrm{C} 10$ & $-2.0(3)$ & $\mathrm{C} 13-\mathrm{C} 14-\mathrm{C} 15-\mathrm{N} 2$ & $0.10(19)$ \\
\hline $\mathrm{F} 1-\mathrm{C} 9-\mathrm{C} 10-\mathrm{C} 11$ & $-178.83(15)$ & $\mathrm{C} 19-\mathrm{C} 14-\mathrm{C} 15-\mathrm{N} 2$ & $177.52(16)$ \\
\hline $\mathrm{C} 8-\mathrm{C} 9-\mathrm{C} 10-\mathrm{C} 11$ & $1.4(3)$ & $\mathrm{C} 14-\mathrm{C} 13-\mathrm{C} 16-\mathrm{C} 17$ & $11.8(3)$ \\
\hline $\mathrm{F} 1-\mathrm{C} 9-\mathrm{C} 10-\mathrm{N} 2$ & $1.2(2)$ & $\mathrm{C} 12-\mathrm{C} 13-\mathrm{C} 16-\mathrm{C} 17$ & $-170.41(17)$ \\
\hline $\mathrm{C} 8-\mathrm{C} 9-\mathrm{C} 10-\mathrm{N} 2$ & $-178.51(16)$ & $\mathrm{C} 13-\mathrm{C} 16-\mathrm{C} 17-\mathrm{C} 18$ & $-40.6(2)$ \\
\hline $\mathrm{C} 15-\mathrm{N} 2-\mathrm{C} 10-\mathrm{C} 9$ & $-108.0(2)$ & $\mathrm{C} 16-\mathrm{C} 17-\mathrm{C} 18-\mathrm{C} 19$ & $60.9(2)$ \\
\hline $\mathrm{C} 12-\mathrm{N} 2-\mathrm{C} 10-\mathrm{C} 9$ & $60.9(2)$ & $\mathrm{C} 13-\mathrm{C} 14-\mathrm{C} 19-\mathrm{C} 18$ & $17.6(3)$ \\
\hline $\mathrm{C} 15-\mathrm{N} 2-\mathrm{C} 10-\mathrm{C} 11$ & $72.1(2)$ & $\mathrm{C} 15-\mathrm{C} 14-\mathrm{C} 19-\mathrm{C} 18$ & $-159.46(17)$ \\
\hline $\mathrm{C} 12-\mathrm{N} 2-\mathrm{C} 10-\mathrm{C} 11$ & $-119.05(19)$ & $\mathrm{C} 17-\mathrm{C} 18-\mathrm{C} 19-\mathrm{C} 14$ & $-46.4(2)$ \\
\hline
\end{tabular}

Hydrogen-bond geometry $\left(A,{ }^{\circ}\right)$

$\mathrm{Cg} 1$ is the centroid of the $\mathrm{C} 3 / \mathrm{C} 4 / \mathrm{C} 8-\mathrm{C} 11$ ring.

\begin{tabular}{lllll}
\hline$D-\mathrm{H} \cdots A$ & $D-\mathrm{H}$ & $\mathrm{H} \cdots A$ & $D \cdots A$ & $D-\mathrm{H}^{\cdots} \cdots A$ \\
\hline $\mathrm{C} 7-\mathrm{H} 7 \cdots \mathrm{O}^{\mathrm{i}}$ & 0.95 & 2.39 & $3.178(2)$ & 140 \\
$\mathrm{C} 19-\mathrm{H} 19 B \cdots \mathrm{F} 1^{\mathrm{ii}}$ & 0.99 & 2.36 & $3.289(2)$ & 155 \\
$\mathrm{C} 16-\mathrm{H} 16 A \cdots C g 1^{\mathrm{iii}}$ & 0.99 & 2.63 & $3.5601(19)$ & 157 \\
\hline
\end{tabular}

Symmetry codes: (i) $x+1, y, z$; (ii) $x, y-1, z$; (iii) $-x+2, y-1 / 2,-z+1 / 2$. 